\title{
CSRR based patch antenna for Wi-Fi and WiMAX Applications
}

\author{
Suman Nelaturi ${ }^{1}$, NVSN Sarma ${ }^{2}$ \\ ${ }^{1,2}$ Department of ECE, National Institute of Technology Warangal, Telangana, India \\ *corresponding author, E-mail: nelaturi.suman4@gmail.com
}

\begin{abstract}
In this paper, a novel compact microstrip patch antenna is proposed for $\mathrm{Wi}-\mathrm{Fi}$ and WiMAX bands. To achieve miniaturization the dimensions of the square radiating patch are chosen with reference to the high frequency band (3.3 $\mathrm{GHz}$ ). The dual band is achieved by loading a Complementary Split Ring Resonator (CSRR) into the radiating patch. The left handed nature of the CSRR is the cause for low frequency band $(2.4 \mathrm{GHz})$. To improve the return loss bandwidth and axial ratio bandwidth at upper band the fractal concept is introduced along the edges of the square patch. Thus a low volume dual band antenna is simulated using HFSS. A comparison with measured data is also presented. The fabricated antenna is found to be occupying $25 \%$ less volume (with reference to $2.4 \mathrm{GHz}$ ) than existing antennas which is mainly due to the blending of the two recent concepts 'metamaterials and fractals'.
\end{abstract}

\section{Introduction}

The modern wireless hand held gadget need to operate at multiple bands to be able to provide communication as well as GPS types of other services [1]. Instead of using multiple antennas for multiple operating frequencies, a single Microstrip Patch Antenna (MPA) which serves at all operating frequencies like WLAN, Wi-Fi and WiMAX applications with less size and wider bandwidth at each operating frequency is the most wanted candidate for today's wireless hand held devices. In conventional dual band or multiband MPAs the dimensions of the radiating patch are taken with reference to lowest frequency band which requires more size, however in the case of dual band antennas based on metamaterials the dimensions of the radiating patch are taken with reference to high frequency band which requires less size because of left handed nature. The novelty of this antenna is to embed metamaterials and fractal curves into the conventional MPA to realize at Wi-Fi and WiMAX applications.

Metamaterials are classified into Epsilon Negative materials (ENG), Permeability Negative materials (MNG), and Double Negative materials (DNG) [2, 3]. These materials can be realized by using metallic , Split Ring Resonators (SRRs) and the combination of both Vias and SRRs respectively. The characteristics of SRRs and CSRRs have been studied and analyzed by several groups [4- 17]. By applying the concept of duality to the negative permeability nature of SRR, the negative permittivity nature of CSRR can be obtained. The CSRR unit cell has strong potential applications in designing $\mathrm{CP}$ antennas and dual band antennas because of its special property of zero mode resonance. In [5-8], dual band antennas are presented by inserting CSRR in the radiating patch. In [9- 13], single band and dual band antennas are designed by etching CSRR from the ground plane. In [14- 16], dual band and single band antennas are designed by using MNG materials. In [17], the combination of both DNG and Double Positive materials is used to get dual band antenna. The antennas mentioned above occupy a large volume in terms of surface area or thickness for Wi-Fi and WiMAX applications and also provide low bandwidth. However, with thin sized hand held devices becoming popular, there is a need for designing an ultra thin antenna to operate at $3.4 \mathrm{GHz}$ and $2.4 \mathrm{GHz}$ with wide bandwidth at each frequency. To obtain $\mathrm{CP}$ with a single feed arrangement at patch mode (High frequency) the fractal concept is introduced into the symmetrical radiating patch $[18,19]$.

In this paper, with a single layer patch antenna is proposed using both poly fractal concept as well as CSRR for the first time. The probe feed mechanism is adopted for its ease of use. Ansoft HFSS (High Frequency Structure Simulator) software tool is used to simulate the proposed antenna. The measurement with the fabricated antenna is compared with the simulated data.

\section{Geometry of the proposed antenna}

The geometry of the proposed antenna is shown in the Fig.1. Rogers RT/Duroid 5880 substrate with dielectric constant 2.2 and thickness $3.175 \mathrm{~mm}$ is used. CSRR is etched from the patch to get dual band operation. The dimensions of the proposed antenna are listed in Table 1.

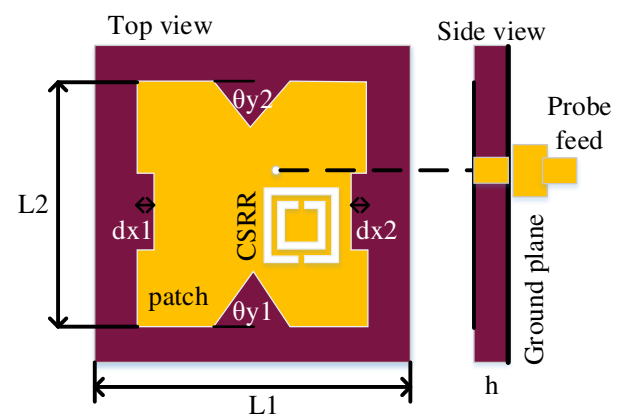

Figure 1: Geometry of the proposed antenna 
Table 1: This is an example of a table.

\begin{tabular}{cc}
\hline Parameter & Value in $\mathrm{mm}$ \\
\hline L1 & 37 \\
L2 & 27 \\
h & 3.175 \\
a & 10 \\
b & 10 \\
c & 9 \\
d & 9 \\
g1 & 0.4 \\
g2 & 0.4 \\
g3 & 0.4 \\
dx1=dx2 & 1 \\
dy1=dy2 & 3.5 \\
\hline
\end{tabular}

\subsection{CSRR and fractal curves}

The two topologies of Split Ring Resonator (SRR) and CSRR are shown in the Fig. 2. According to Babinet's principle CSRR is the dual structure of the SRR in which the roles of metal and air are interchanged, and also the role of electric and magnetic field components. The SRR can act as magnetic dipole that can be excited by an external magnetic flux where the CSRR can act as electric dipole that can be excited by external electric field. The equivalent circuit diagram of SRR and CSRR are shown in the Fig. 3, where the $\mathrm{C} 0$ is the total capacitance between the rings, LO is the total inductance, Ls is the series inductance of the $\mathrm{SRR}, \mathrm{Lc}$ is the shunt inductance, $\mathrm{Cc}$ is the shunt capacitance and $\mathrm{Cs}$ is the series capacitance of the lower and upper halves of the SRR. The resonant frequency of the SRR is given by

$f_{0}=\frac{1}{2 \pi\left(C_{s} L_{s}\right)}$

Based on duality concept of SRR and CSRR the resonant frequency of CSRR is same as the resonant frequency of SRR.

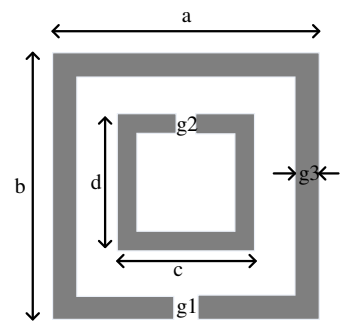

a

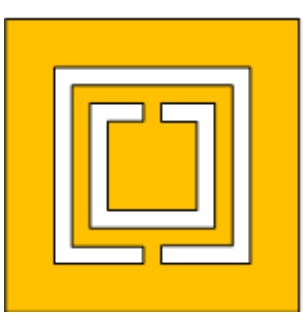

b
Figure 2: Topology of a SRR and b CSRR

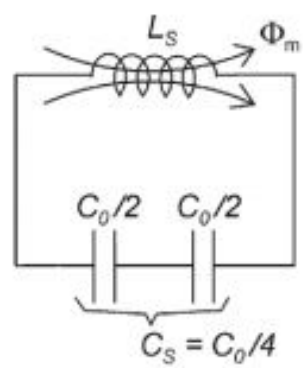

a

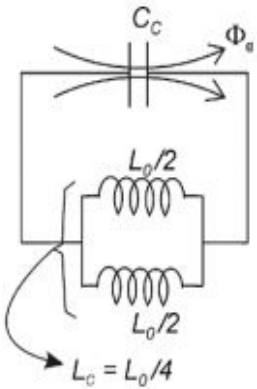

$\mathrm{b}$
Figure 3: Equivalent circuit model of a SRR b CSRR

Fractal curves are self repetitive and space filling curves which are used to design compact antennas. Fractal curves are characterized by two parameters: Iteration Order (IO) and Indentation Factor (IF). The IF is different for different types of fractals. For example, the IF for the Minkowski fractal curve is Indentation Depth (ID) where Dx and Dy are the IDs along $\mathrm{x}$ - and $\mathrm{y}$ - axes, and for the Koch fractal curves the same is Indentation Angle (IA) where $\theta x$ and $\theta y$ are the IAs along $\mathrm{x}$ - and $\mathrm{y}$ - axes. The IAs of Koch fractal curves are shown in the Fig. 4.

In the case of Koch fractal curves, the equal IA $(\theta \times 1=\theta \times 2=\theta y 1=\theta y 2)$ along four sides of the square patch antenna results in LP at upper frequency band. CP radiation can be obtained by placing different IAs along each side of the patch $(\theta \mathrm{x} 1 \neq \theta \mathrm{x} 2 \neq \theta \mathrm{y} 1 \neq \theta \mathrm{y} 2)$ or $(\theta \mathrm{x} 1=\theta \mathrm{x} 2=\theta \mathrm{x} \neq \theta \mathrm{y} 1=$ $\theta y 2=\theta y)$ where the high bandwidth can be obtained when IAs along same axis are equal and are another along the perpendicular axis.

In the case of Minkowski fractal curves, the equal ID (Dx1=Dx2=Dy1=Dy2) along four sides of the square patch antenna results in linear polarization (LP) at upper frequency band. CP radiation can be obtained by loading different IDs along each side of the square patch (Dx $1 \neq$ Dx $2 \neq$ Dy $1 \neq$ Dy 2$)$ or $(D x 1=$ Dx $2=$ Dx $\neq$ Dy $1=$ Dy $2=$ Dy $)$ where the high bandwidth can be obtained when IDs along same axis are equal and are not equal along the perpendicular axis.

The poly fractal boundary patch antenna can be obtained by using the combination of both Koch and Minkowski fractal curves. The edges of the square patch are replaced by Koch fractal curve along $\mathrm{X}^{-}$axis and Minkowski fractal curve along $y$-axis. The IDs and IAs of poly fractal boundary patch are shown in the Fig. 3 .

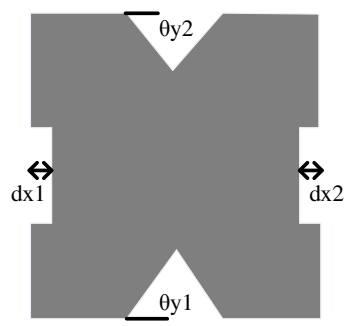

Figure 4: IDs and IAs of poly fractal boundary patch 


\section{Simulation Results}

The evolution of the proposed design can be understood by considering an evolution of patch antenna design shown in Fig. 5.

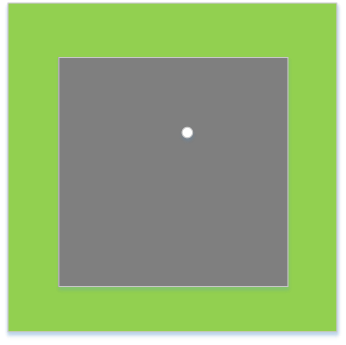

Ant1

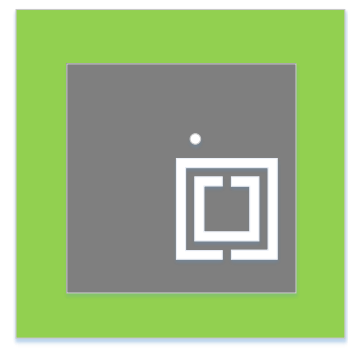

Ant3

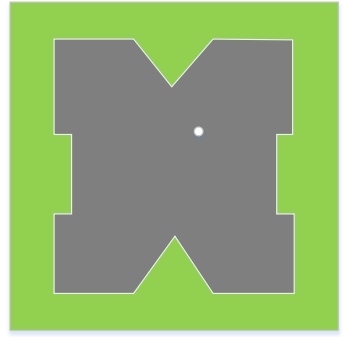

Ant2

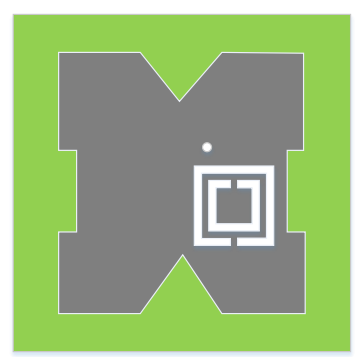

Ant4
Figure 5: Evolution of the proposed antenna

Initially, the square patch Ant1 is chosen as a reference which is resonating at $3.4 \mathrm{GHz}$ with linear polarization. Poly fractal curves are introduced along edges of the square patch Ant 1 to get Ant 2 which results in CP. Ant3 can be obtained by introducing CSRR in the patch which results in double band operation with Linear Polarization. Ant4 represents the dual band dual polarization version achieved by introducing poly fractal curves to the edges of Ant3. Finally, Ant 4 is the proposed antenna with dual band dual polarization.

The simulated return loss characteristics of all proposed antennas are shown in the Fig. 6. By loading CSRR in the radiating patch, the dual band dual polarization operation is obtained. The circular polarization bandwidth at patch mode can be achieved by inserting poly fractal curves along the edges of the patch. The 10-dB return loss bandwidth of all resonating frequencies is listed in Table 2.

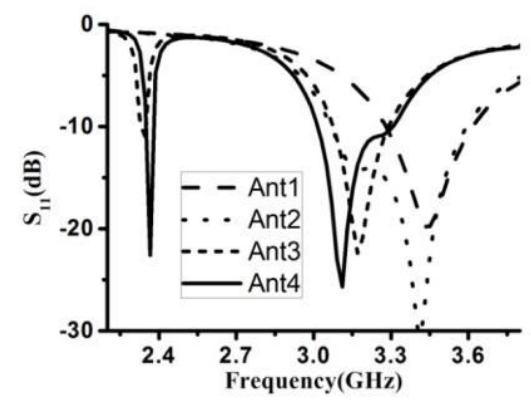

Figure 6: Return loss characteristics of the proposed antennas

Table 2: Impedance bandwidth of simulated antennas.

\begin{tabular}{|c|c|c|}
\hline \multirow[t]{2}{*}{ Antenna } & \multicolumn{2}{|c|}{$10-\mathrm{dB}$ return loss bandwidth } \\
\hline & $\begin{array}{l}\text { at left hand } \\
\text { band }\end{array}$ & at patch mode band \\
\hline Ant1 & --- & $\begin{array}{c}9.27 \%(3.29-3.61 \\
\mathrm{GHz})\end{array}$ \\
\hline Ant 2 & --- & $\begin{array}{c}15.06 \%(3.07-3.57 \\
\mathrm{GHz})\end{array}$ \\
\hline Ant3 & $\begin{array}{c}\text { Resonance at } \\
2.4 \mathrm{GHz}\end{array}$ & $\begin{array}{c}5.66 \%(3.09-3.27 \\
\mathrm{GHz})\end{array}$ \\
\hline Ant4 & $\begin{array}{c}\text { Resonance at } \\
2.4 \mathrm{GHz}\end{array}$ & $\begin{array}{c}8.22 \%(3.03-3.29 \\
\mathrm{GHz})\end{array}$ \\
\hline
\end{tabular}

To understand the operational mechanism of the proposed antenna, the HFSS simulated current distribution on patch Ant 4 at each frequency band is shown in the Fig. 7.
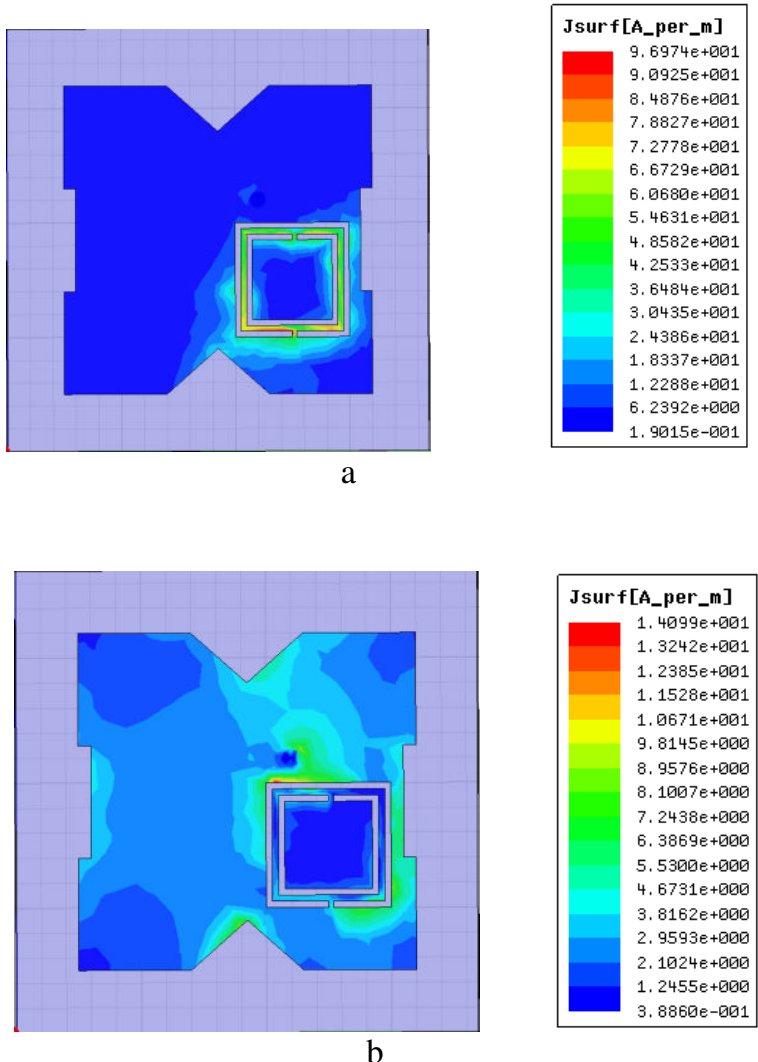

Figure 7: Simulated Surface current distribution at a 2.4 $\mathrm{GHz}$ b $3.3 \mathrm{GHz}$.

The lower resonating frequency $(2.4 \mathrm{GHz})$ is mainly due to strong surface current distribution at CSRR where as the upper resonating frequency $(3.3 \mathrm{GHz})$ is due to current at poly fractal edges of the patch. The simulated radiation efficiency characteristics of the proposed antenna are shown in the Figure 8. The efficiency at 2.4 $\mathrm{GHz}$ is $96.05 \%$ which is less compared to efficiency value of $98.75 \%$ at $3.3 \mathrm{GHz}$. 


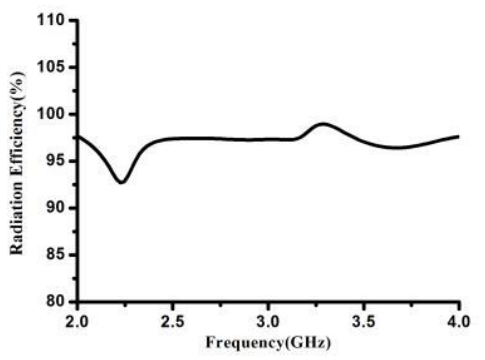

Figure 8: Radiation efficiency of the proposed antenna

\section{Measured Results}

The proposed antenna (Ant4) fabricated on Rogers RT/Duroid substrate with dimensions $37 \times 37 \times 3.175 \mathrm{~mm} 3$ is shown in Fig. 8. The return loss characteristics are measured using Agilent 8719A microwave network analyzer. Radiation pattern measurements are taken in an anechoic chamber having the physical dimensions of 22.5 $\mathrm{x} 12.5 \times 11.5 \mathrm{~m} 3$ and with the operating frequency range of $400 \mathrm{MHz}$ to $18 \mathrm{GHz}$.

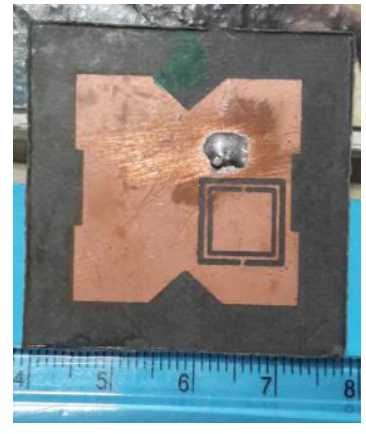

a

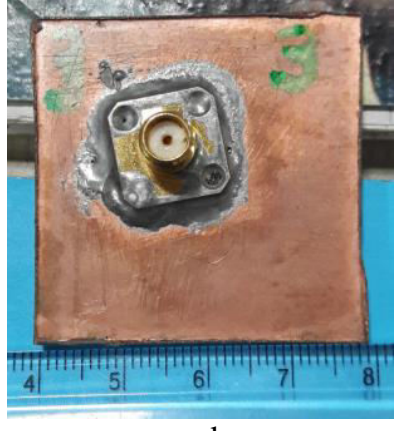

b
Figure 8: Fabricated prototype a top view b bottom view

The measured return loss characteristics along with simulated data are given in Fig. 9. Measured results are in close agreement with simulated results. The discontinuity at upper resonating band indicates the possibility of circular polarization radiation.

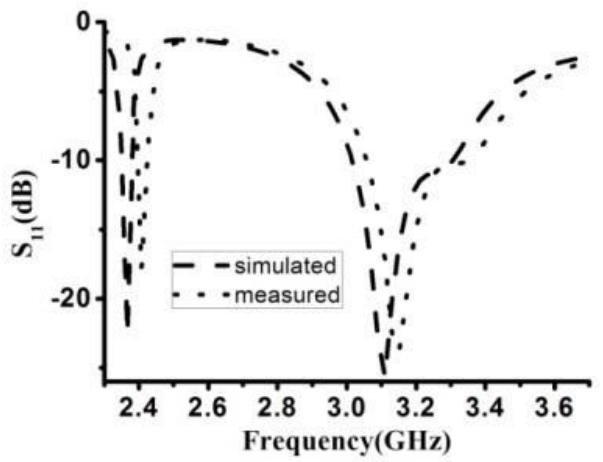

Figure 9: Return loss characteristics of the fabricated antenna

The 10-dB return loss bandwidth is $8.22 \%$ (3.01- 3.27 $\mathrm{GHz}$ ) at upper resonating frequency band due to current at patch edges and $<1 \%$ (resonance at $2.4 \mathrm{GHz}$ only) at lower left handed resonance frequency band because of current at CSRR respectively. The Axial ratio characteristics are given in Fig. 10. The measured 3-dB axial ratio bandwidth at the upper resonating frequency is $2.50 \%(3.15-3.23 \mathrm{GHz})$. The minimum Axial Ratio value of $0.3 \mathrm{~dB}$ occurred at the center frequency.

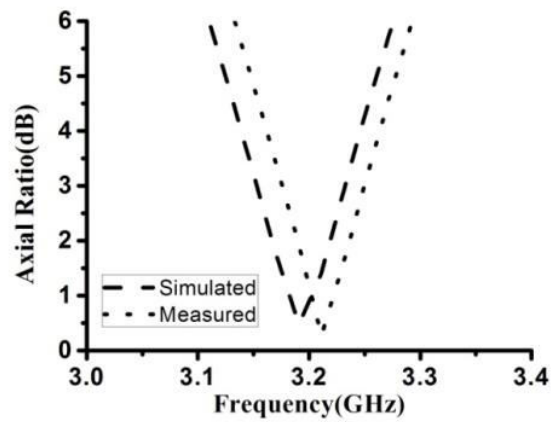

Figure 10: Axial Ratio characteristics of the proposed antenna

The radiation patterns of the proposed antenna at the lower and upper resonating bands $(2.4 \mathrm{GHz}$ and 3.2 GHZ) are shown in the Fig. 11\& 12 respectively. The gain of the proposed antenna is given in Fig. 13.
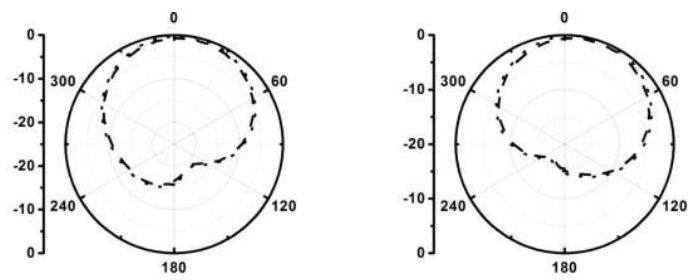

- - Simulated

....Measured

a

b

Figure 11: Simulated and measured radiation characteristics of the proposed antenna at $2.4 \mathrm{GHz}$ a $\mathrm{E}$ plane b H plane
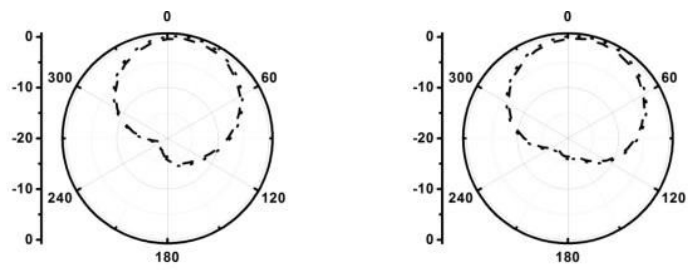

- - Simulated

.... Measured

b

Figure 12: Simulated and measured radiation characteristics of the proposed antenna at $3.2 \mathrm{GHz}$ a $\mathrm{E}$ plane b H plane 


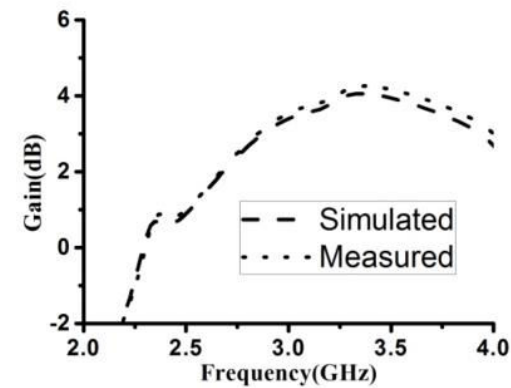

Figure 13: Gain characteristics of the proposed antenna

Table 3 gives the comparison between the results of the proposed antenna and the antennas which already exist in the literature. The proposed antenna is more compact and producing wide bandwidth compared to remaining antennas listed in Table 3.

Table 3: Comparison with existing literature.

\begin{tabular}{|c|c|c|c|c|}
\hline \multirow[t]{2}{*}{ Ref } & \multirow[t]{2}{*}{$\begin{array}{c}\text { Antenna } \\
\text { size }\left(\mathrm{mm}^{2}\right)\end{array}$} & \multirow[t]{2}{*}{$\begin{array}{c}\text { Substrate } \\
\text { size }\left(\mathrm{mm}^{3}\right)\end{array}$} & \multicolumn{2}{|c|}{$\begin{array}{c}\text { Resonant } \\
\text { frequency } \\
(\mathrm{GHz}), \\
\text { 10-dB return } \\
\text { loss } \\
\text { bandwidth } \\
(\%)\end{array}$} \\
\hline & & & $\begin{array}{c}\text { At } \\
\text { lower } \\
\text { band }\end{array}$ & $\begin{array}{c}\text { At } \\
\text { upper } \\
\text { band }\end{array}$ \\
\hline prop & $27 \times 27$ & $37 \times 37 \times 3.175$ & $2.4,--$ & $\begin{array}{l}3.2 \\
8.22\end{array}$ \\
\hline [5] & $18.43 \times 23.68$ & & $4.1,-$ & $4.8,-$ \\
\hline [6] & $18.43 \times 23.68$ & & $4.1,-$ & $4.8,-$ \\
\hline [7] & $18.43 \times 23.68$ & & $4.1,-$ & $4.8,-$ \\
\hline [8] & 16.8 & $32.62 \times 3$ & $1.6,-$ & $4.5,-$ \\
\hline [9] & $20 \times 16$ & $30 \times 50 \times 1.6$ & $2.9,-$ & $3.6,-$ \\
\hline [10] & & $58 \times 23 \times 1.5$ & $\begin{array}{c}0.917 \\
1\end{array}$ & $\begin{array}{c}1.854, \\
1.5\end{array}$ \\
\hline [11] & $17.7 \times 17.5$ & & $\begin{array}{c}3.73, \\
1.2\end{array}$ & $\begin{array}{c}5.25 \\
1.5\end{array}$ \\
\hline [12] & $19 \times 19$ & $40 \times 35 \times 1.57$ & \multicolumn{2}{|c|}{$6.8,--$} \\
\hline [13] & $8 \times 8$ & $\begin{array}{l}28 \times 28 \times 0.762 \\
28 \times 28 \times 0.762\end{array}$ & \multicolumn{2}{|c|}{$5.15,--$} \\
\hline$[14]$ & $40 \times 40$ & $80 \times 80 \times 5$ & $0.4,-$ & $2.4,-$ \\
\hline [15] & $5 \times 0.5$ & & \multicolumn{2}{|c|}{$9.51,--$} \\
\hline [16] & 16.8 & $32.62 \times 3$ & $1.62,-$ & $\begin{array}{c}4.53, \\
-\end{array}$ \\
\hline [17] & $16.8 \times 20.8$ & $40 \times 50 \times 0.49$ & $4.9,--$ & $5.3,-$ \\
\hline
\end{tabular}

\section{Conclusion}

A novel miniaturized single probe feed poly fractal boundary patch antenna loaded with CSRR for dual band operation has been presented. The Simulated $10-\mathrm{dB}$ return loss bandwidth of proposed antenna is $<1 \%$ (resonating at $2.4 \mathrm{GHz}$ only) at the lower resonating frequency and is $8.72 \%(3.07-3.35 \mathrm{GHz})$ at the upper resonating frequency. The 3-dB axial ratio bandwidth of the antenna at upper frequency band is around 2.49\% (3.17- 3.25 $\mathrm{GHz}$ ). Proposed antenna can be conveniently used in portable devices because of its compactness for $\mathrm{Wi}-\mathrm{Fi}$ and WiMAX applications.

\section{References}

[1] Garg, Ramesh. Microstrip antenna design handbook. Artech House, 2001. ISBN 0-89006513-6.

[2] Eleftheriades, George V., and Keith G. Balmain. Negative-refraction metamaterials: fundamental principles and applications. John Wiley \& Sons, 2005.

[3] Caloz, Christophe, and Tatsuo Itoh. Electromagnetic metamaterials: transmission line theory and microwave applications. John Wiley \& Sons, 2005.

[4] Marqués, Ricardo, et al. "Comparative analysis of edge-and broadside-coupled split ring resonators for metamaterial design-theory and experiments." IEEE Transactions on Antennas and Propagation 51.10 (2003): 2572-2581.

[5] Ortiz, N., F. Falcone, and M. Sorolla. "Dual band patch antenna based on complementary rectangular split-ring resonators." Microwave Conference, 2009. APMC 2009. Asia Pacific. IEEE, 2009.

[6] Ortiz, N., F. Falcone, and M. Sorolla. "Enhanced gain dual band patch antenna based on complementary rectangular split-ring resonators." Microwave and Optical Technology Letters 53.3 (2011): 590-594.

[7] Ortiz, Noelia, Francisco Falcone, and Mario Sorolla. "Gain improvement of dual band antenna based on complementary rectangular split-ring resonator." ISRN Communications and Networking 2012 (2012): 3.

[8] Ortiz, Noelia, et al. "Design and implementation of dual-band antennas based on complementary split ring resonators." Waves in Random and Complex Media 25.3 (2015): 309-322.

[9] Lee, Yoonjae, and Yang Hao. "Characterization of microstrip patch antennas on metamaterial substrates loaded with complementary split-ring 
resonators." Microwave and Optical Technology Letters 50.8 (2008): 2131-2135.

[10] Niu, J-X. "Dual-band dual-mode patch antenna based on resonant-type metamaterial transmission line." Electronics letters 46.4 (2010): 266-268.

[11] Xie, Yihong, et al. "A novel dual-band patch antenna with complementary split ring resonators embedded in the ground plane." progress in electromagnetics research letters 25 (2011): 117-126.

[12] Ha, Jaegeun, et al. "Hybrid mode wideband patch antenna loaded with a planar metamaterial unit cell." IEEE Transactions on Antennas and Propagation 60.2 (2012): 1143-1147.

[13]Ramzan, Mehrab, and Kagan Topalli. "A miniaturized patch antenna by using a CSRR loading plane." International Journal of Antennas and Propagation 2015 (2015).

[14] Bilotti, Filiberto, Andrea Alu, and Lucio Vegni. "Design of Miniaturized Metamaterial Patch Antennas With $\mu$-Negative Loading." IEEE Transactions on Antennas and Propagation 56.6 (2008): 1640-1647.

[15]Joshi, J. G., et al. "Electrically small patch antenna loaded with metamaterial." IETE Journal of Research 56.6 (2010): 373-379.

[16] Tang, Ming-Chun, et al. "A Compact Dual-Band Patch Antenna Design Based on Single-Ring Split Ring Resonator." Applied Computational Electromagnetics Society Journal 31.3 (2016).

[17] Xiong, Jiang, et al. "Modified $\mathrm{TM}_{020}$ Mode of a Rectangular Patch Antenna Partially Loaded With Metamaterial for Dual-Band Applications." IEEE Antennas and wireless Propagation letters 8 (2009): 1006-1009.

[18] Barnsley, Michael F. Fractals everywhere. Academic press, 2014.

[19] Reddy, V. V., and N. V. S. N. Sarma. "Poly Fractal Boundary Circularly Polarized Microstrip Antenna for WLAN/Wi-MAX Wireless Applications." Defense Science Journal 65.5 (2015): 379-384. 